\title{
LIMITS OF THE MULTIPLIER ADJUSTMENT APPROACH TO CAPACITATED LOCATION PROBLEM
}

This contribution deals with a distribution system design problem, in which the located facilities satisfy all customer demands under limited abilities. The objective is to minimize the total costs, including both fixed charges and service costs. A special approach based on Lagrangean relaxation will be discussed here for its ability to cope with limited capacities of located facilities. Using the Lagrangean relaxation, the model of the original problem is rearranged to a model, which can be solved by exact algorithms for considerably large size. The capacities of the located facilities bring serious difficulties concerning solving technique, in the cases, where real-sized facility location problems are solved. In contrast to an uncapacitated facility location problem, which can be solved exactly in reasonable time for real-sized case containing hundreds of possible locations and thousands of customers.

In this paper, we shall discuss a transformation of the capacitated location problem into the uncapacitated location problem by means of Lagrangean relaxation of capacity constraints. To demonstrate the efficiency of the studied approach, numerical experiments were performed and their results are reported in the concluding part of this paper.

\section{Introduction}

A distribution system can be considered as a sort of a transportation system, which enables delivering goods from one or several sources to customers. This delivering can be either direct or with transhipment at several places (these are generally called terminals or facilities) - see Fig. 1.



Fig. 1. Design of a distribution system

Determination of structure of a distribution system with transhipment belongs to the family of location problems. The associated location problem can be formulated as follows: given a set of possible facility locations and a set of customers, which are served from a finite number of located facilities, find which facilities should be used and which customers should be served from which facilities so that to minimise the total cost of serving all the customers and the fixed investment costs of building and maintaining the facilities.
In this paper, we shall investigate the capacitated location problem, with limited annual capacities of individual facilities. We shall devise the appropriate mathematical model and the solution method.

The capacitated location problem can be formulated as $0-1$ linear programming problem, but the searching of its exact solution is very time-consuming or nearly impossible for large cases. One of possible approaches to a capacitated location problem can be based on its rearrangement to the uncapacitated location problem, solution of which can be found relatively fast, also for real-life size.

\section{A Model of the Capacitated Location Problem}

As preliminaries for a model construction we introduce the following notation of particular terms, which will be used throughout the whole paper.

Let $J$ denote a finite set of customers and if a quantity of customer's demand can be expressed by a real number, then the demand of customer $j \in J$ is denoted by $b_{j}$.

Let $I$ denote a finite set of possible facility locations, than the decision on a facility location at place $i \in I$ is modelled by zeroone variable $y_{i} \in\{0,1\}$, which takes value 1 if a facility should be located at $i$ and it takes value 0 otherwise.

The model of the problem can be formulated as follows:

$$
\text { Minimize } \sum_{i \in I} f_{i} y_{i}+\sum_{i \in I} \sum_{j \in J} c_{i j} z_{i j}
$$

\footnotetext{
* Lýdia Gábrišová

Department of Mathematical Methods, Faculty of Management and Informatics Science, University of Žilina, Univerzitná 1, 01026 Žilina, Slovak Republic, Tel. +421-41-5134 285, E-mail: gabriska@frcatel.fri.utc.sk
} 


$$
\begin{array}{lll}
\text { Subject to } & \sum_{i \in I} z_{i j}=1 & \text { for } j \in J \\
& y_{i}-z_{i j} \geq 0 & \text { for } i \in I, j \in J \\
& \sum_{j \in J} b_{j} z_{i j} \leq a_{i} & \text { for } i \in I \\
& y_{i} \in\{0,1\} & \text { for } i \in I \\
& z_{i j} \in\{0,1\} & \text { for } i \in I, j \in J
\end{array}
$$

In this model $f_{i}$ denotes the fixed investment and maintenance cost of the facility with location $i \in I$ in the considered time period.

$c_{i j}$ is the cost of demand satisfaction of customer $j \in J$ via the facility $i \in I$. Size of the demand is $b_{j}$ and $\operatorname{cost} c_{i j}$ can be modelled as follows:

$$
c_{i j}=\left(e_{0} d_{s i}+e_{1} d_{i j}+g_{i}\right) b_{j}
$$

where $d_{s i}$ is the distance between a primary source s and a facility $i, d_{i j}$ is the distance between a terminal (facility location) $i$ and a customer $j, e_{1}$ denotes unit transportation costs on the link between the primary source $s$ and the facility $i, e_{0}$ denotes unit transportation costs on the link between the facility $i$ and the customer $j$ and $g_{i}$ denotes the unit cost for the reloading of goods in the facility location $i$.

We suppose that the capacity of a facility located at $i$ is $a_{i}$.

Constraints (2) ensure that each customer demand must be satisfied from exactly one facility location and constraints (3) force out the placement of a facility at location $i$ whenever a customer is assigned to this facility location. Constraints (4) ensure that the total demand satisfied via facility $i$ does not exceed the given capacity $a_{i}$.

If constrains (4) are omitted, the problem (1) - (3), (5), (6) is known as the uncapacitated location problem.

\section{A Solving method for the Capacitated Location Problem}

For the exact solving of an uncapacitated facility location problem (1) - (3), (5) - (6), the suitable solution method is the branch-and-bound method with branching performed by fixing the chosen variable $y_{i}$ to the value 0 or 1 in the depth-first search of the solution tree.

We shall use the branch-and-bound method for the exact solution of the uncapacitated location problem based on Erlenkotter's approach [Erlenkotter, 1978], which was modified as BBDual algorithm by [Janáček, Kováčiková, 1997]. The solution yields the number of used facilities, their spatial distribution and the association of the customers with the facilities.

Next, let us show how we can reduce the capacitated location problem to the uncapacitated one by means of Lagrangean relax- ation. Let us introduce the vector $u$ of non-negative Lagrangean multipliers, $u_{i} \geq 0$ for all $i \in I$ and rearrange the objective function (1) to the following form:

$$
\begin{aligned}
& \sum_{i \in I} f_{i} y_{i}+\sum_{i \in I} \sum_{j \in J} c_{i j} z_{i j}+\sum_{i \in I} u_{i}\left(\sum_{j \in J} b_{j} z_{i j}-a_{i}\right)= \\
& =\sum_{i \in I} f_{i} y_{i}+\sum_{i \in I} \sum_{j \in J} c_{i j} z_{i j}+\sum_{i \in I} \sum_{j \in J} u_{i} b_{j} z_{i j}-\sum_{i \in I} u_{i} a_{i}= \\
& =\sum_{i \in I} f_{i} y_{i}+\sum_{i \in I} \sum_{j \in J} z_{i j}\left(c_{i j}+u_{i} b_{j}\right)-\sum_{i \in I} u_{i} a_{i} .
\end{aligned}
$$

By omitting the last term of expression (7), we obtain the following expression:

$$
\sum_{i \in I} f_{i} y_{i}+\sum_{i \in I} \sum_{j \in J} c_{i j} z_{i j}
$$

where $\quad \underline{c}_{i j}=c_{i j}+u_{i} b_{j}$

for $i \in I, j \in J$.

The last term in (7) is a constant for fixed $u_{i}$, and so we can omit it when solving the reformulated problem. Substituting the optimal solution of (8), (2), (3), (5), (6) into (7), we obtain a lower bound of the objective function value of a so-far unknown optimal solution of (1) - (6). Moreover, if a solution of (8), (2), (3), (5), (6) satisfies constraints (4) and complementarity constraints (9), then this solution is the optimal solution of the capacitated problem.

$$
u_{i}\left(\sum_{j \in J} b_{j} z_{i j}-a_{i}\right)=0 \text { for } i \in I
$$

In the other case, we can use this solution as a starting point of a dual heuristics.

To obtain an approximate solution of the capacitated location problem we start with the relaxed problem model, Lagrangean multipliers of which are set to zero. If the optimal solution of this problem obtained by BBDual algorithm satisfies capacity constrains (4), then the optimal solution of the original problem is found.

Otherwise (if capacities of some of the facilities are not sufficient for the given demands), we change multiplier values. We make the changes by choosing the facility sequentially with the maximal difference between capacity and total demand of the associated customers and solve the relaxed problem with adjusted multipliers by BBDual algorithm.

An increase of the multiplier associated with a given facility should result in decrease of the capacity deficit associated with the facility. The stopping criterion for our algorithm was that the summary deficit of all located facilities should be less than $5 \%$ of the summary demand of all customers, i.e.:

$$
\begin{aligned}
& \sum_{i \in I_{k}}\left(\sum_{j \in J} b_{j} z_{i j}-a_{i}\right) \leq \sum_{j \in J} b_{j} / 100 * 5 \text { where } I_{k} \subseteq I, \\
& I_{k}=\left\{i \in I \sum_{j \in J} b_{j} z_{i j}>a_{i}\right\} .
\end{aligned}
$$




\section{Experiments}

Lagrangean relaxation of the capacitated location problem was implemented in Delphi and real data from the Slovak road network was used for numerical experiments. In the solved problems, 71 regional towns form the set of possible facility locations and 2907 villages form the set of customers. Demands of customers depend on the number of inhabitants in the villages. The costs connected with serving customers from a located facility are derived from distances in the real road network. The computations were performed with various values of fixed costs and capacities of facilities.

In most cases, a change in fixed costs causes a change in the number and locations of facilities, which can increase if the values of multipliers increase. This raise of the number of facilities may cause that all customer's demands are satisfied unless the facility capacities are exceeded (see Table 1).

The experiments showed that the change of the multiplier (increment by 1) could cause increase of the objective function (1), and we can obtain a new solution.

In Table 2 there is an example in which the sum of all customer demands is 53808 and the first selection of 10 facilities has a total capacity of 53900. Increase of u4 to 4 decreased the sum of all deficit demands from $7.98 \%$ to $3.22 \%$ and added 1 facility. Other changes of multiplier values in the above-mentioned way do not decrease the sum of deficit demands.

Location problem for various capacity of a facilities

Table 1

\begin{tabular}{|c|c|c|c|c|c|c|c|c|}
\hline \multirow[t]{2}{*}{ number of steps } & \multirow[t]{2}{*}{ located facilities } & \multicolumn{2}{|c|}{$\begin{array}{l}\text { values of } \\
\text { multiplier }\end{array}$} & \multirow[t]{2}{*}{$\begin{array}{l}\text { objective function } \\
\text { (1) }\end{array}$} & \multirow[t]{2}{*}{$\begin{array}{l}\text { objective function } \\
\text { (7) }\end{array}$} & \multirow{2}{*}{$\begin{array}{c}\begin{array}{c}\text { not covered } \\
\text { customer demands } \\
(\max )\end{array} \\
\end{array}$} & \multirow{2}{*}{$\begin{array}{c}\text { not covered } \\
\text { customer demands } \\
\text { (sum) }\end{array}$} & \multirow[t]{2}{*}{$\%$} \\
\hline & & 4 & 47 & & & & & \\
\hline \multicolumn{9}{|c|}{ Capacity of 2 facilities: } \\
\hline 0 & 2 & 0 & 0 & 17865901 & 17865901 & 7795 & 7795 & 14.49 \\
\hline 1 & 2 & 1 & & 17865901 & 17873696 & 7795 & 7795 & 14.49 \\
\hline 2 & 2 & 2 & & 17866076 & 17881316 & 7620 & 7620 & 14.16 \\
\hline 3 & 2 & 3 & & 17866076 & 17888936 & 7620 & 7620 & 14.16 \\
\hline 4 & 2 & 4 & & 17986996 & 17986996 & 7592 & 7592 & 14.11 \\
\hline 5 & 2 & & 1 & 17866076 & 17896556 & 7620 & 7620 & 14.16 \\
\hline 6 & 2 & 5 & & 17986996 & 17994574 & 7578 & 7578 & 14.08 \\
\hline 7 & 3 & & 2 & 17943985 & 17886395 & 0 & 0 & 0.00 \\
\hline \multicolumn{9}{|c|}{ Capacity of 2 facilities: } \\
\hline 0 & 2 & 0 & 0 & 17865901 & 17865901 & 13098 & 13098 & 24.34 \\
\hline 1 & 2 & 1 & & 17865901 & 17878999 & 13098 & 13098 & 24.34 \\
\hline 2 & 2 & 2 & & 17866076 & 17891922 & 12923 & 12923 & 24.02 \\
\hline 3 & 2 & 3 & & 17866076 & 17904845 & 12923 & 12923 & 24.02 \\
\hline 4 & 2 & 4 & & 17986996 & 17986996 & 12895 & 12895 & 23.96 \\
\hline 5 & 2 & & 1 & 17866076 & 17917768 & 12923 & 12923 & 24.02 \\
\hline 6 & 2 & 5 & & 17986996 & 17999877 & 12881 & 12881 & 23.94 \\
\hline 7 & 3 & & 2 & 17943985 & 17912910 & 0 & 0 & 0.00 \\
\hline
\end{tabular}

Location problem for 53900 capacity of 10 facilities

Table 2

\begin{tabular}{|c|c|c|c|c|c|c|c|c|c|}
\hline \multirow[t]{2}{*}{ number of steps } & \multirow[t]{2}{*}{ located facilities } & \multicolumn{3}{|c|}{$\begin{array}{l}\text { values of } \\
\text { multiplier }\end{array}$} & \multirow[t]{2}{*}{$\begin{array}{l}\text { objective function } \\
\text { (1) }\end{array}$} & \multirow[t]{2}{*}{$\begin{array}{l}\text { objective function } \\
\text { (7) }\end{array}$} & \multirow{2}{*}{$\begin{array}{c}\text { not covered } \\
\text { customer demands } \\
(\max )\end{array}$} & \multirow{2}{*}{$\begin{array}{c}\text { not covered } \\
\text { customer demands } \\
\text { (sum) }\end{array}$} & \multirow[t]{2}{*}{$\%$} \\
\hline & & 4 & 47 & & & & & & \\
\hline 0 & 10 & 0 & 0 & 0 & 11684833 & 11684833 & 2833 & 4295 & 7.98 \\
\hline 1 & 10 & 1 & & & 11684837 & 11687666 & 2829 & 4291 & 7.97 \\
\hline 2 & 10 & 2 & & & 11684843 & 11690489 & 2823 & 4285 & 7.96 \\
\hline 3 & 10 & 3 & & & 11684876 & 11693312 & 2812 & 4274 & 7.94 \\
\hline 4 & 11 & 4 & & & 11693307 & 11694387 & 1063 & 1732 & 3.22 \\
\hline
\end{tabular}




\begin{tabular}{|c|c|c|c|c|c|c|c|c|c|}
\hline \multirow[t]{2}{*}{ number of steps } & \multirow[t]{2}{*}{ located facilities } & \multicolumn{3}{|c|}{$\begin{array}{l}\text { values of } \\
\text { multiplier }\end{array}$} & \multirow[t]{2}{*}{$\begin{array}{l}\text { objective function } \\
\text { (1) }\end{array}$} & \multirow[t]{2}{*}{$\begin{array}{l}\text { objective function } \\
\text { (7) }\end{array}$} & \multirow{2}{*}{$\begin{array}{c}\text { not covered } \\
\text { customer demands } \\
(\max )\end{array}$} & \multirow{2}{*}{$\begin{array}{c}\text { not covered } \\
\text { customer demands } \\
(\text { sum })\end{array}$} & \multirow[t]{2}{*}{$\%$} \\
\hline & & 4 & 47 & 36 & & & & & \\
\hline 0 & 9 & 0 & 0 & 0 & 12058775 & 12058775 & 4275 & 6772 & 12.59 \\
\hline 1 & 9 & 1 & & & 12058798 & 12063050 & 4252 & 6772 & 12.59 \\
\hline 13 & 9 & 13 & & & 12060284 & 12112440 & 4012 & 6772 & 12.59 \\
\hline 14 & 9 & 14 & & & 12060479 & 12116437 & 3997 & 6765 & 12.57 \\
\hline 20 & 10 & 20 & & & 12118942 & 12136922 & 1965 & 5276 & 9.81 \\
\hline 21 & 10 & & 1 & & 12121718 & 12139598 & 1925 & 4492 & 8.35 \\
\hline 22 & 10 & & & 1 & 12118944 & 12138887 & 1963 & 5294 & 9.84 \\
\hline 23 & 10 & & 2 & & 12121618 & 12141523 & 1925 & 4500 & 8.36 \\
\hline 24 & 10 & & & 2 & 12118990 & 12140804 & 1917 & 5253 & 9.76 \\
\hline 31 & 10 & & 6 & & 12123534 & 12147239 & 1145 & 3766 & 7.00 \\
\hline 32 & 10 & & & 6 & 12111701 & 12147163 & 1471 & 3940 & 7.32 \\
\hline 33 & 10 & 21 & & & 12246944 & 12256388 & 1574 & 5007 & 9.31 \\
\hline 34 & 10 & & 7 & & 12250594 & 12257464 & 1145 & 3550 & 6.60 \\
\hline \multirow[t]{2}{*}{35} & 10 & & & 7 & 12294238 & 12294238 & 2429 & 4630 & 8.60 \\
\hline & & 42 & & & & & & & \\
\hline 36 & 10 & 1 & & & 12251266 & 12257363 & 871 & 2164 & 4.02 \\
\hline
\end{tabular}

If we choose different input fixed costs in the testing instance, we can obtain 9 facilities with a total capacity of 48510 . That is less than $90.16 \%$ of total demands. The first selection of the facilities caused total difference of $12.59 \%$. Successive increase of multipliers decreased the total difference to $4.02 \%$ after 36 steps, but caused the raise of the number of facilities to 10 (see Table 3 ).

\section{Conclusions}

The Lagrangean relaxation seems to be an effective solving method for some cases of capacitated location problems with limited annual capacities of individual located facilities. If the capacity constraints need not be satisfied exactly and values of multipliers are successively increased for a located facility with the maximal number of deficit demands then the numerical experiments in most cases give acceptable results.

Acknowledgement: This paper was supported by the institutional grant of Faculty of Management and Informatics Science, University of Žilina: 19/2005 and VEGA No.1/0490/03.

\section{References}

[1] JANÁČEK, J.: Mathematical Programming (in Czech), EDIS - ŽU, Žilina, 2003, p. 225 s.

[2] JANÁČEK, J.: Optimisation in Transportation Networks (in Czech), EDIS - ŽU, Žilina, 2003, p. 248

[3] DREZNER, ZVI (ed.) et al.: Facility location, Applications and theory, Berlin, Springer Verlag, 2002, ISBN 3-540-42172-6.

[4] JANÁČEK, J.: Service System Design in The Public and Private Sector, In Proceedings of the International Conference: Quantitative Methods in Economics (Multiple Criteria Decision Making XII), June 2-4, 2004 Virt, Slovak Republic, pp. 101 $\div 108$

[5] BUZNA, L.: Distribution System Structure Design Using Continuous Approximation and Discrete Programming (in Slovak), PhD-Thesis, FRI, ŽU, Žilina, 2003, p. 90. 